\title{
Robust performance of virtual sensing methods for active noise control
}

\author{
Jin Zhang, Stephen J. Elliott, Jordan Cheer
}

Institute of Sound and Vibration Research, University of Southampton SO17 1BJ, United Kingdom

\begin{abstract}
This paper investigates the effect of changes in the environment on the performance of two widelyused virtual sensing methods for active noise control (ANC): the remote-microphone method and the additional-filter method. Robust performance of adaptive feedforward control algorithms incorporating such virtual sensing techniques is essential to achieving noise attenuation at the designated locations in practice, when subject to uncertainties in the control environment. Off-line simulations using the data measured with a headrest ANC system in a running car are initially conducted, to evaluate the performance of the two virtual sensing methods under practical conditions. The differences between the two methods are further studied by using an analytical model and numerical simulations of the headrest ANC system. It is shown that in general the additional-filter method is sensitive to uncertainties in the properties of the reference signals used for feedforward control, whereas the remote-microphone method is sensitive to changes in the plant responses related to the monitoring microphones. This study, therefore, can be used to guide the choice of virtual sensing methods in different applications.
\end{abstract}

Keywords: Active noise control, Virtual sensing, Robust performance, In-car measurement

\section{Introduction}

Active noise control (ANC) systems combined with virtual sensing techniques can achieve noise reduction at locations where the physical sensors cannot be placed [1, 2. A popular practical example is the headrest ANC system, which aims to attenuate the noise at the listener's ears in 5 a vehicle $3-5$. The techniques of virtual sensing initially started by using off-line modelling to achieve noise reduction at the designated locations, although the performance of such systems is inevitably restricted by the accuracy of the modelling [6]. Improvements were made by introducing a preliminary identification phase, during which physical microphones are placed at the locations of the virtual error microphones [2]. This procedure aims to obtain a design containing the information about the system, which will later be used to obtain the control filter to realise control of the noise disturbance at the virtual microphones when the physical microphones are removed. The position of the virtual sensors can also be adapted, using head tracking for example Refs. 2, 7].

Email addresses: jz1a19@soton.ac.uk (Jin Zhang), S.J.Elliott@soton.ac.uk (Stephen J. Elliott), J.Cheer@soton.ac.uk (Jordan Cheer) 
Two main methods have been suggested for virtual sensing in ANC systems. One is known as the remote-microphone (RM) method [2, 6, 8], which provides an estimate of the noise disturbance at the virtual error microphones from the disturbances at the monitoring microphones via an observation filter. Analyses in terms of both numerical simulations and practical experiments have been conducted to investigate the RM method [911. The results of these studies show that the $\mathrm{RM}$ method can achieve noise reduction over a wide frequency range, up to about $1 \mathrm{kHz}$ in the laboratory. Later tests using measurements from a running vehicle also demonstrate the practical noise cancellation capability of this method 9 .

Another method, which is called the additional-filter (AF) method in this paper, is fundamentally a model reference adaptive control strategy [1, 12]. This method is realised by initially minimising the noise at error microphones placed at the virtual sensor positions. An additional filter is then trained to model the responses from the reference signals to the monitoring micro-

${ }_{25}$ phones when the signals at the virtual sensor locations have been minimised. During the control phase, the adaptive controller minimises the difference between the monitoring microphones and the output of the additional filter. The latest measurements with the implementation of the AF method provide promising outcomes in terms of noise attenuation at the virtual microphones [13].

Due to the fundamentally different natures of these two methods, their performances will differ, especially when there are perturbations in the environment. An understanding of this would potentially instruct the future implementations of the virtual sensing techniques to achieve the optimal noise reduction. In this paper, the formulations of the two virtual sensing methods are presented in Sec. 2. In Sec. 3. measured data recorded in the cabin of a running car is utilised to simulate the performance of the virtual sensing methods in reducing the noise at the ears of

35 a dummy head. By manipulating the measured data, the robust performance of the two virtual sensing methods under different conditions is simulated. A simple numerical model of a headrest ANC system is presented in Sec. 4, in which perturbations can be systematically introduced. A combination of analytical formulations and numerical simulations is used to explain the differences between the results obtained from the measurements. The conclusions are presented in Sec. 5 . 40 where guidelines for future implementations of the virtual sensing methods are given.

\section{The virtual sensing methods}

In this section, the two virtual sensing methods, the remote microphone (RM) method and the additional filter (AF) method, are explained using a multichannel frequency-domain formulation. All signals are assumed to be stationary random to ensure their spectral densities are time-invariant. 45 For notational simplicity, their frequency dependence is suppressed.

The RM method and the AF method are each assumed to be implemented on the same ANC system, which consists of $N_{x}$ reference sensors, $N_{u}$ secondary sources, $N_{m}$ monitoring microphones and $N_{e}$ virtual error sensors that are placed at the designated location(s) for noise reduction. Multichannel signals are denoted as lower case bold vectors, e.g. $\mathbf{m}$ and e for the signals of the 
monitoring and error sensors, and the matrices of frequency responses are denoted as upper case bold matrices, e.g. $\mathbf{G}_{\mathrm{m}}$ and $\mathbf{G}_{\mathrm{e}}$ for the responses form the secondary sources to the monitoring and error sensors. The two virtual sensing methods can both be divided into two phases: the identification (tuning or training) phase and the control phase. In the first phase, physical microphones are temporarily placed at the locations of the virtual error sensors. Two filters are obtained by utilising

55 these physical error sensors, the observation filter $\mathbf{O}$ for the RM method and the additional filter $\hat{\mathbf{H}}$ for the AF method, where $(\hat{\cdot})$ denotes the estimates obtained during the identification phase, During the control phase, where the temporarily-placed sensors are removed, these filters are then applied to optimise the feedforward control filter $\mathbf{W}$ to achieve sound reduction at the virtual sensors, which are remote from the physical monitoring sensors.

\subsection{Remote microphone method}

Fig. 1 shows the block diagram of the RM method during the identification and control phases. The observation filter $\hat{\mathbf{O}}$, as shown in Fig. 1(a), is the identified transfer matrix between the primary disturbances measured at the monitoring sensors $\hat{\mathbf{d}}_{\mathrm{m}}=\hat{\mathbf{P}}_{\mathrm{m}} \hat{\mathbf{v}}$ and at the error sensors $\hat{\mathbf{d}}_{\mathrm{e}}=\hat{\mathbf{P}}_{\mathrm{e}} \hat{\mathbf{v}}_{\text {, }}$ where $\hat{\mathbf{v}}$ denotes the primary sources at the identification phase, and $\hat{\mathbf{P}}_{\mathrm{m}}$ and $\hat{\mathbf{P}}_{\mathrm{e}}$ are the matrices of responses from the primary sources to the monitoring sensors and error microphones respectively during the identification phase. The least square estimate of $\hat{\mathbf{O}}$ can be straightforwardly obtained by minimising the cost function,

$$
J_{\hat{\mathbf{o}}}=\operatorname{Tr}\left\{E\left[\left(\hat{\mathbf{d}}_{\mathrm{e}}-\hat{\mathbf{O}} \hat{\mathbf{d}}_{\mathrm{m}}\right)\left(\hat{\mathbf{d}}_{\mathrm{e}}-\hat{\mathbf{O}} \hat{\mathbf{d}}_{\mathrm{m}}\right)^{\mathrm{H}}\right]\right\}
$$

where $\operatorname{Tr}\{\cdot\}$ denotes the trace of the matrix, $E\{\cdot\}$ denotes the expectation operator and $(\cdot)^{\mathrm{H}}$ denotes the conjugate transpose. As stated in Ref. [9], the observation filter $\hat{\mathbf{O}}$ could be ill-conditioned, and, therefore, may require an addition regularisation term in Eq. (1), which leads to

$$
J_{\hat{\mathbf{O}}}=\operatorname{Tr}\left\{E\left[\left(\hat{\mathbf{d}}_{\mathrm{e}}-\hat{\mathbf{O}} \hat{\mathbf{d}}_{\mathrm{m}}\right)\left(\hat{\mathbf{d}}_{\mathrm{e}}-\hat{\mathbf{O}} \hat{\mathbf{d}}_{\mathrm{m}}\right)^{\mathrm{H}}+\beta_{\mathrm{RM}} \hat{\mathbf{O}} \hat{\mathbf{O}}^{\mathrm{H}}\right]\right\}
$$

where $\beta_{\mathrm{RM}}$ is the regularisation factor for $\hat{\mathbf{O}}$. The optimal observation filter $\hat{\mathbf{O}}_{\mathrm{Opt}}$ is, hence [10, 14], given by

$$
\begin{aligned}
\hat{\mathbf{O}}_{\mathrm{Opt}} & =\mathbf{S}_{\hat{\mathbf{d}}_{\mathrm{m}} \hat{\mathbf{d}}_{\mathrm{e}}}\left(\mathbf{S}_{\hat{\mathbf{d}}_{\mathrm{m}} \hat{\mathbf{d}}_{\mathrm{m}}}+\beta_{\mathrm{RM}} \mathbf{I}\right)^{-1} \\
& =\hat{\mathbf{P}}_{\mathrm{e}} \mathbf{S}_{\hat{\mathbf{v}} \hat{\mathbf{v}}} \hat{\mathbf{P}}_{\mathrm{m}}^{\mathrm{H}}\left(\hat{\mathbf{P}}_{\mathrm{m}} \mathbf{S}_{\hat{\mathbf{v}} \hat{\mathbf{v}}} \hat{\mathbf{P}}_{\mathrm{m}}^{\mathrm{H}}+\beta_{\mathrm{RM}} \mathbf{I}\right)^{-1}
\end{aligned}
$$

where $\mathbf{S}_{\hat{\mathbf{v}} \hat{\mathbf{v}}}=E\left[\hat{\mathbf{v}} \hat{\mathbf{v}}^{\mathrm{H}}\right]$ is the power spectral density matrix of $\hat{\mathbf{v}}, \mathbf{S}_{\hat{\mathbf{d}}_{\mathrm{m}} \hat{\mathbf{d}}_{\mathrm{e}}}=E\left[\hat{\mathbf{d}}_{\mathrm{e}} \hat{\mathbf{d}}_{\mathrm{m}}^{\mathrm{H}}\right]$ is the cross spectral density matrix between $\hat{\mathbf{d}}_{\mathrm{m}}$ and $\hat{\mathbf{d}}_{\mathrm{e}}, \mathbf{S}_{\hat{\mathbf{d}}_{\mathrm{m}} \hat{\mathbf{d}}_{\mathrm{m}}}=E\left[\hat{\mathbf{d}}_{\mathrm{m}} \hat{\mathbf{d}}_{\mathrm{m}}^{\mathrm{H}}\right]$ is the power spectral density matrix of $\hat{\mathbf{d}}_{\mathrm{m}}$ and $\mathbf{I}$ is an identity matrix that has the same dimensions as $\mathbf{S}_{\hat{\mathbf{d}}_{\mathrm{m}} \hat{\mathbf{d}}_{\mathrm{m}}}$. The inclusion of the regularisation term can improve the robustness of the method against uncertainties, but this comes with a trade-off in terms of the ultimate noise attenuation capability [5]. In addition, $\hat{\mathbf{G}}_{\mathrm{m}}$ and $\hat{\mathbf{G}}_{\mathrm{e}}$, which are the estimates of the plant responses from the secondary sources to both the monitoring sensors and the error sensors, are also obtained during the identification phase. 
During the control phase, in Fig. 1(b), the signals at the monitoring sensors $\mathbf{m}$ are due to both the primary disturbances $\mathbf{d}_{\mathrm{m}}=\mathbf{P}_{\mathrm{m}} \mathbf{v}$ and the secondary sources $\mathbf{u}_{\mathrm{RM}}=\mathbf{W}_{\mathrm{RM}} \mathbf{x}$, can be written as

$$
\mathbf{m}=\mathbf{d}_{\mathrm{m}}+\mathbf{G}_{\mathrm{m}} \mathbf{u}_{\mathrm{RM}}
$$

where $\mathbf{P}_{\mathrm{m}}$ and $\mathbf{G}_{\mathrm{m}}$ are the matrices of the primary paths and secondary paths to the monitoring sensors during the control phase. Similarly, v denotes the primary sources during the control phase and $\mathbf{x}$ is the vector of the reference signals used to represent the primary field in practice. $\mathbf{x}$ can be expressed in terms of $\mathbf{v}$ as

$$
\mathbf{x}=\mathbf{R} \mathbf{v}
$$

where $\mathbf{R}$ is the matrix of responses between primary sources, $\mathbf{v}$ and the reference signals. If the reference signals provide an accurate representation of all the primary sources, then $\mathbf{R}$ would be a simple identity matrix. As shown in Fig. 1(b), an estimate of the primary disturbances, $\tilde{\mathbf{d}}_{\mathrm{m}}$, can be obtained by subtracting an estimate of the secondary disturbance from $\mathbf{m}$. Under nominal condition, the estimation error for the RM method is then [5]

$$
\boldsymbol{\epsilon}=\mathbf{d}_{\mathrm{e}}-\hat{\mathbf{O}}_{\mathrm{Opt}} \mathbf{d}_{\mathrm{m}}
$$

This can be considered as representative of the spatial correlation between the monitoring sensors and the error sensors and indicates the lower limit on the error signals at the virtual microphones that can be obtained with the RM method. The estimate of the primary disturbance at the virtual error sensors, $\tilde{\mathbf{d}}_{\mathrm{e}}$, can consequently be obtained by using $\hat{\mathbf{O}}_{\mathrm{Opt}}$ in Fig. 1(b). Combining $\tilde{\mathbf{d}}_{\mathrm{e}}$ with the estimated contribution of the secondary sources, $\hat{\mathbf{G}}_{\mathrm{m}} \mathbf{u}_{\mathrm{RM}}$, the estimated sound pressure at the virtual error sensors can be obtained as

$$
\begin{aligned}
\tilde{\mathbf{e}} & =\tilde{\mathbf{d}}_{\mathrm{e}}+\hat{\mathbf{G}}_{\mathrm{e}} \mathbf{u}_{\mathrm{RM}} \\
& =\hat{\mathbf{O}}_{\mathrm{Opt}}\left(\mathbf{m}-\hat{\mathbf{G}}_{\mathrm{m}} \mathbf{u}_{\mathrm{RM}}\right)+\hat{\mathbf{G}}_{\mathrm{e}} \mathbf{u}_{\mathrm{RM}}
\end{aligned}
$$

By substituting Eq. (4) into Eq. (7),

$$
\tilde{\mathbf{e}}=\hat{\mathbf{O}}_{\mathrm{Opt}} \mathbf{d}_{\mathrm{m}}+\mathbf{G}_{\mathrm{RM}} \mathbf{W}_{\mathrm{RM}} \mathbf{x}
$$

where $\mathbf{G}_{\mathrm{RM}}$, the matrix of the effective plant responses, is given by

$$
\mathbf{G}_{\mathrm{RM}}=\hat{\mathbf{G}}_{\mathrm{e}}+\hat{\mathbf{O}}_{\mathrm{Opt}}\left(\mathbf{G}_{\mathrm{m}}-\hat{\mathbf{G}}_{\mathrm{m}}\right)
$$

The optimal control filter $\mathbf{W}_{\mathrm{RM} \text {,Opt }}$ can be obtained by minimising the cost function of $J_{\tilde{\mathbf{e}}}=$ $\operatorname{Tr}\left\{E\left[\tilde{\mathbf{e}} \tilde{\mathbf{e}}^{\mathrm{H}}\right]\right\}$ to give [10, 15]

$$
\begin{aligned}
\mathbf{W}_{\mathrm{RM}, \mathrm{Opt}} & =-\left[\mathbf{G}_{\mathrm{RM}}^{\mathrm{H}} \mathbf{G}_{\mathrm{RM}}\right]^{-1} \mathbf{G}_{\mathrm{RM}}^{\mathrm{H}} \hat{\mathbf{O}}_{\mathrm{Opt}} \mathbf{S}_{\mathbf{x} \mathbf{d}_{\mathrm{m}}} \mathbf{S}_{\mathbf{x x}}^{-1} \\
& =-\left[\mathbf{G}_{\mathrm{RM}}^{\mathrm{H}} \mathbf{G}_{\mathrm{RM}}\right]^{-1} \mathbf{G}_{\mathrm{RM}}^{\mathrm{H}} \hat{\mathbf{O}}_{\mathrm{Opt}} \mathbf{P}_{\mathrm{m}} \mathbf{S}_{\mathbf{v v}} \mathbf{R}^{\mathrm{H}}\left(\mathbf{R} \mathbf{S}_{\mathbf{v v}} \mathbf{R}^{\mathrm{H}}\right)^{-1}
\end{aligned}
$$

In practice, however, $\mathbf{W}_{\mathrm{RM}, \mathrm{Opt}}$ is calculated by adaptation using the filtered-reference LMS (FxLMS) algorithm [14, in which the reference signal is filtered by $\hat{\mathbf{G}}_{\mathrm{e}}$, as obtained in the identification phase. 
The converged control filter then becomes Ref. [15]

$$
\begin{aligned}
\mathbf{W}_{\mathrm{RM}, \infty} & =-\left[\hat{\mathbf{G}}_{\mathrm{e}}^{\mathrm{H}} \mathbf{G}_{\mathrm{RM}}\right]^{-1} \hat{\mathbf{G}}^{\mathrm{H}} \hat{\mathbf{O}}_{\mathrm{Opt}} \mathbf{S}_{\mathbf{x d}} \mathbf{S}_{\mathbf{x x}}^{-1} \\
& =-\left[\hat{\mathbf{G}}_{\mathrm{e}}^{\mathrm{H}} \mathbf{G}_{\mathrm{RM}}\right]^{-1} \hat{\mathbf{G}}_{\mathrm{e}}^{\mathrm{H}} \hat{\mathbf{O}}_{\mathrm{Opt}} \mathbf{P}_{\mathrm{m}} \mathbf{S}_{\mathbf{v v}} \mathbf{R}^{\mathrm{H}}\left(\mathbf{R S}_{\mathbf{v v}} \mathbf{R}^{\mathrm{H}}\right)^{-1} .
\end{aligned}
$$

A sufficient convergence condition for the adaptive algorithm is that [7]

$$
\operatorname{Re}\left(\operatorname{eig}\left[\hat{\mathbf{G}}_{\mathrm{e}}^{\mathrm{H}} \mathbf{G}_{\mathrm{RM}}\right]\right)=\operatorname{Re}\left(\operatorname{eig}\left[\hat{\mathbf{G}}_{\mathrm{e}}^{\mathrm{H}} \hat{\mathbf{G}}_{\mathrm{e}}+\hat{\mathbf{G}}_{\mathrm{e}}^{\mathrm{H}} \mathbf{O}_{\mathrm{Opt}}\left(\mathbf{G}_{\mathrm{m}}-\hat{\mathbf{G}}_{\mathrm{m}}\right)\right]\right)>0
$$

By using Eqs. (9) and (11), the signal that would actually be measured at the virtual error sensors if the control filter converged to the solution given by Eq. (11) can be obtained as

$$
\begin{aligned}
\mathbf{e}_{\mathrm{RM}}=\mathbf{d}_{\mathrm{e}}+\mathbf{G}_{\mathrm{e}} \mathbf{W}_{\mathrm{RM}, \infty} \mathbf{x} & =\mathbf{d}_{\mathrm{e}}-\mathbf{G}_{\mathrm{e}}\left\{\hat{\mathbf{G}}_{\mathrm{e}}^{\mathrm{H}}\left[\hat{\mathbf{G}}_{\mathrm{e}}+\hat{\mathbf{O}}_{\mathrm{Opt}}\left(\mathbf{G}_{\mathrm{m}}-\hat{\mathbf{G}}_{\mathrm{m}}\right)\right]\right\}^{-1} \hat{\mathbf{G}}_{\mathrm{e}} \hat{\mathbf{O}}_{\mathrm{Opt}} \times \\
& \mathbf{P}_{\mathrm{m}} \mathbf{S}_{\mathbf{v v}} \mathbf{R}^{\mathrm{H}}\left(\mathbf{R} \mathbf{S}_{\mathbf{v v}} \mathbf{R}^{\mathrm{H}}\right)^{-1} \mathbf{R v} . \\
& \longrightarrow \widehat{\mathbf{P}}_{\widehat{\mathbf{v}}} \\
& \longrightarrow \widehat{\mathbf{P}}_{\mathrm{m}}
\end{aligned}
$$

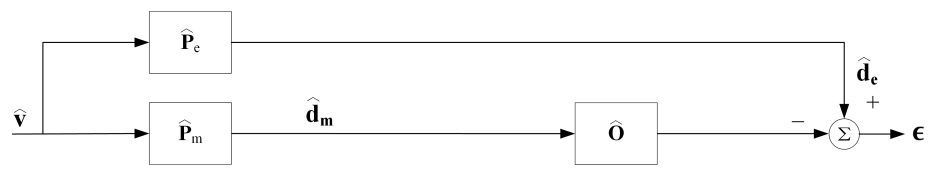

(a)

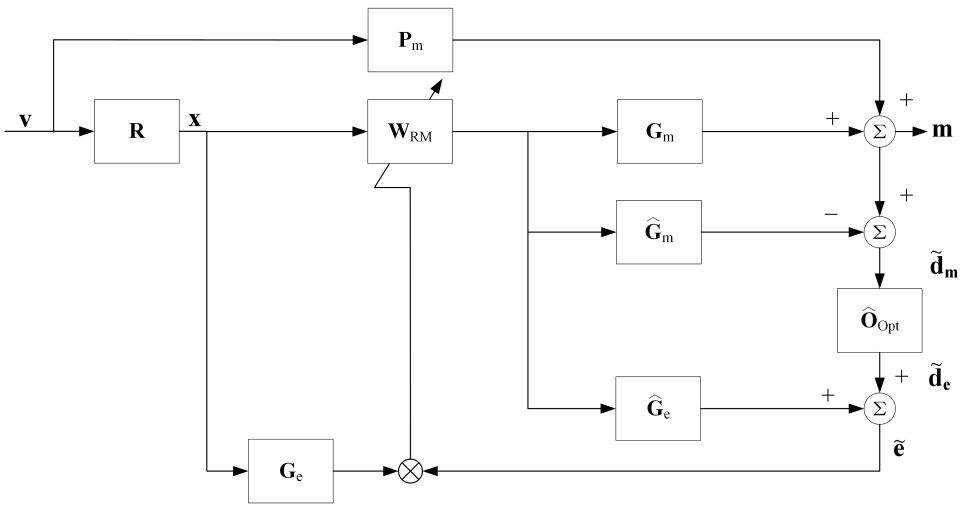

(b)

Figure 1: The block diagram of the RM method: (a) identification phase; (b) control phase.

\subsection{Additional filter method}

Fig. 2(a) shows the block diagram of the AF method during the identification phase. This phase includes the training of two filters, an initial control filter $\hat{\mathbf{W}}_{\mathrm{AF}}$, designed to minimise the mean square error signals, and the additional filter $\hat{\mathbf{H}}$, designed to model the response from the reference signals to the monitoring signals once the error signals have been controlled. The control 
filter $\hat{\mathbf{W}}_{\mathrm{AF}}$ is only utilised to drive the secondary sources during the identification phase, but its response is then implicit in the additional filter $\hat{\mathbf{H}}$. $\hat{\mathbf{W}}_{\mathrm{AF}, \text { Opt }}$ can be obtained by minimising the signals at the error sensors, $\hat{\mathbf{e}}$, which corresponds to solving the cost function

$$
J_{\hat{\mathbf{W}}_{\mathrm{AF}}}=\operatorname{Tr}\left\{E\left[\left(\hat{\mathbf{d}}_{\mathrm{e}}-\hat{\mathbf{G}}_{\mathrm{e}} \hat{\mathbf{W}}_{\mathrm{AF}} \hat{\mathbf{x}}\right)\left(\hat{\mathbf{d}}_{\mathrm{e}}-\hat{\mathbf{G}}_{\mathrm{e}} \hat{\mathbf{W}}_{\mathrm{AF}} \hat{\mathbf{x}}\right)^{\mathrm{H}}\right]\right\}
$$

to give

$$
\begin{aligned}
\hat{\mathbf{W}}_{\mathrm{AF}, \mathrm{Opt}} & =-\left[\hat{\mathbf{G}}_{\mathrm{e}}^{\mathrm{H}} \hat{\mathbf{G}}_{\mathrm{e}}\right]^{-1} \hat{\mathbf{G}}_{\mathrm{e}}^{\mathrm{H}} \mathbf{S}_{\hat{\mathbf{x}} \hat{\mathbf{d}}_{\mathrm{e}}} \mathbf{S}_{\hat{\mathbf{x}} \hat{\mathbf{x}}}^{-1} \\
& =-\left[\hat{\mathbf{G}}_{\mathrm{e}}^{\mathrm{H}} \hat{\mathbf{G}}_{\mathrm{e}}\right]^{-1} \hat{\mathbf{G}}_{\mathrm{e}}^{\mathrm{H}} \hat{\mathbf{P}}_{\mathrm{e}} \mathbf{S}_{\hat{\mathbf{v}} \hat{\mathbf{v}}} \hat{\mathbf{R}}^{\mathrm{H}}\left(\hat{\mathbf{R}} \mathbf{S}_{\hat{\mathbf{v}} \hat{\mathbf{v}}} \hat{\mathbf{R}}^{\mathrm{H}}\right)^{-1},
\end{aligned}
$$

where $\mathbf{S}_{\hat{\mathbf{x}}_{\mathrm{e}}}=E\left[\hat{\mathbf{d}}_{\mathrm{e}} \hat{\mathbf{x}}^{\mathrm{H}}\right]$ is the cross spectral density matrix between reference signals and the primary disturbance at the error sensors. The additional filter, which is used to estimate the signals at the monitoring sensors via the reference signals, is then introduced after the convergence of $\hat{\mathbf{W}}_{\mathrm{AF}}$. Therefore, the optimal additional filter can be obtained by minimising the cost function

$$
J_{\hat{\mathbf{H}}}=\operatorname{Tr}\left\{E\left[(\hat{\mathbf{m}}-\hat{\mathbf{H}} \hat{\mathbf{x}})(\hat{\mathbf{m}}-\hat{\mathbf{H}} \hat{\mathbf{x}})^{\mathrm{H}}\right]\right\}
$$

where $\hat{\mathbf{m}}$ is the vector signals measured at the monitoring sensors, which is given by

$$
\hat{\mathbf{m}}=\hat{\mathbf{d}}_{\mathrm{m}}+\hat{\mathbf{G}}_{\mathrm{m}} \hat{\mathbf{W}}_{\mathrm{AF}, \mathrm{Opt}} \hat{\mathbf{x}}
$$

The optimal solution to Eq. 16 is given as

$$
\begin{aligned}
\hat{\mathbf{H}}_{\mathrm{Opt}} & =\left(\mathbf{S}_{\hat{\mathbf{x}} \hat{\mathbf{d}}_{\mathrm{m}}}+\hat{\mathbf{G}}_{\mathrm{m}} \hat{\mathbf{W}}_{\mathrm{AF}, \mathrm{Opt}} \mathbf{S}_{\hat{\mathbf{x}} \hat{\mathbf{x}}}\right) \mathbf{S}_{\hat{\mathbf{x}} \hat{\mathbf{x}}}^{-1} \\
& =\left(\hat{\mathbf{P}}_{\mathrm{m}}-\hat{\mathbf{G}}_{\mathrm{m}}\left[\hat{\mathbf{G}}_{\mathrm{e}}^{\mathrm{H}} \hat{\mathbf{G}}_{\mathrm{e}}\right]^{-1} \hat{\mathbf{G}}_{\mathrm{e}}^{\mathrm{H}} \hat{\mathbf{P}}_{\mathrm{e}}\right) \mathbf{S}_{\hat{\mathbf{v}} \hat{\mathbf{v}}} \hat{\mathbf{R}}^{\mathrm{H}}\left(\hat{\mathbf{R}} \mathbf{S}_{\hat{\mathbf{v}} \hat{\mathbf{v}}} \hat{\mathbf{R}}^{\mathrm{H}}\right)^{-1} .
\end{aligned}
$$

During the control phase, the sound reduction at the virtual error sensors is achieved by minimising the effective error signals $\underline{\tilde{\mathbf{e}}}_{\mathrm{AF}}$, which is the difference between the measured signals at the monitoring microphones and the estimate of these signals when the error is minimised,

$$
\underline{\tilde{\mathbf{e}}}_{\mathrm{AF}}=\mathbf{m}-\hat{\mathbf{H}}_{\mathrm{Opt}} \mathbf{x}=\mathbf{d}_{\mathrm{m}}+\mathbf{G}_{\mathrm{m}} \mathbf{W}_{\mathrm{AF}} \mathbf{x}-\hat{\mathbf{H}}_{\mathrm{Opt}} \mathbf{x} .
$$

The optimal control filter $\mathbf{W}_{\mathrm{AF}, \mathrm{Opt}}$ that minimises $J_{\mathbf{W}_{\mathrm{AF}}}=\operatorname{Tr}\left\{E\left[\underline{\tilde{\mathbf{e}}}_{\mathrm{AF}} \underline{\tilde{\mathbf{e}}}_{\mathrm{AF}}^{\mathrm{H}}\right]\right\}$ is given by

$$
\begin{aligned}
\mathbf{W}_{\mathrm{AF}, \mathrm{Opt}} & =\left(\mathbf{G}_{\mathrm{m}}^{\mathrm{H}} \mathbf{G}_{\mathrm{m}}\right)^{-1} \mathbf{G}_{\mathrm{m}}^{\mathrm{H}}\left(\hat{\mathbf{H}}_{\mathrm{Opt}} \mathbf{S}_{\mathbf{x x}}-\mathbf{S}_{\mathbf{x d} \mathbf{d}_{\mathrm{m}}}\right) \mathbf{S}_{\mathbf{x x}}^{-1} \\
& =\left(\mathbf{G}_{\mathrm{m}}^{\mathrm{H}} \mathbf{G}_{\mathrm{m}}\right)^{-1} \mathbf{G}_{\mathrm{m}}^{\mathrm{H}}\left[\hat{\mathbf{H}}_{\mathrm{Opt}}-\mathbf{P}_{\mathrm{m}} \mathbf{S}_{\mathbf{v v}} \mathbf{R}^{\mathrm{H}}\left(\mathbf{R} \mathbf{S}_{\mathbf{v v}} \mathbf{R}^{\mathrm{H}}\right)^{-1}\right] .
\end{aligned}
$$

In practice, however, $\mathbf{W}_{\mathrm{AF}, \mathrm{Opt}}$ is obtained by the adaption of the FxLMS algorithm in Fig. 2(b) with the reference signals filtered by $\hat{\mathbf{G}}_{\mathrm{m}}$, in which case the converged control filter $\mathbf{W}_{\mathrm{AF}, \infty}$ is $[14$ ]

$$
\mathbf{W}_{\mathrm{AF}, \infty}=\left(\hat{\mathbf{G}}_{\mathrm{m}}^{\mathrm{H}} \mathbf{G}_{\mathrm{m}}\right)^{-1} \hat{\mathbf{G}}_{\mathrm{m}}^{\mathrm{H}}\left[\hat{\mathbf{H}}_{\mathrm{Opt}}-\mathbf{P}_{\mathrm{m}} \mathbf{S}_{\mathbf{v v}} \mathbf{R}^{\mathrm{H}}\left(\mathbf{R} \mathbf{S}_{\mathbf{v v}} \mathbf{R}^{\mathrm{H}}\right)^{-1}\right] .
$$

A sufficient the condition for the convergence of $\mathbf{W}_{\mathrm{AF}, \infty}$ is that

$$
\operatorname{Re}\left(\operatorname{eig}\left[\hat{\mathbf{G}}_{\mathrm{m}}^{\mathrm{H}} \mathbf{G}_{\mathrm{m}}\right]\right)>0
$$


The combined contribution of the primary sources and the optimally-controlled secondary sources at the virtual error sensors is then given by

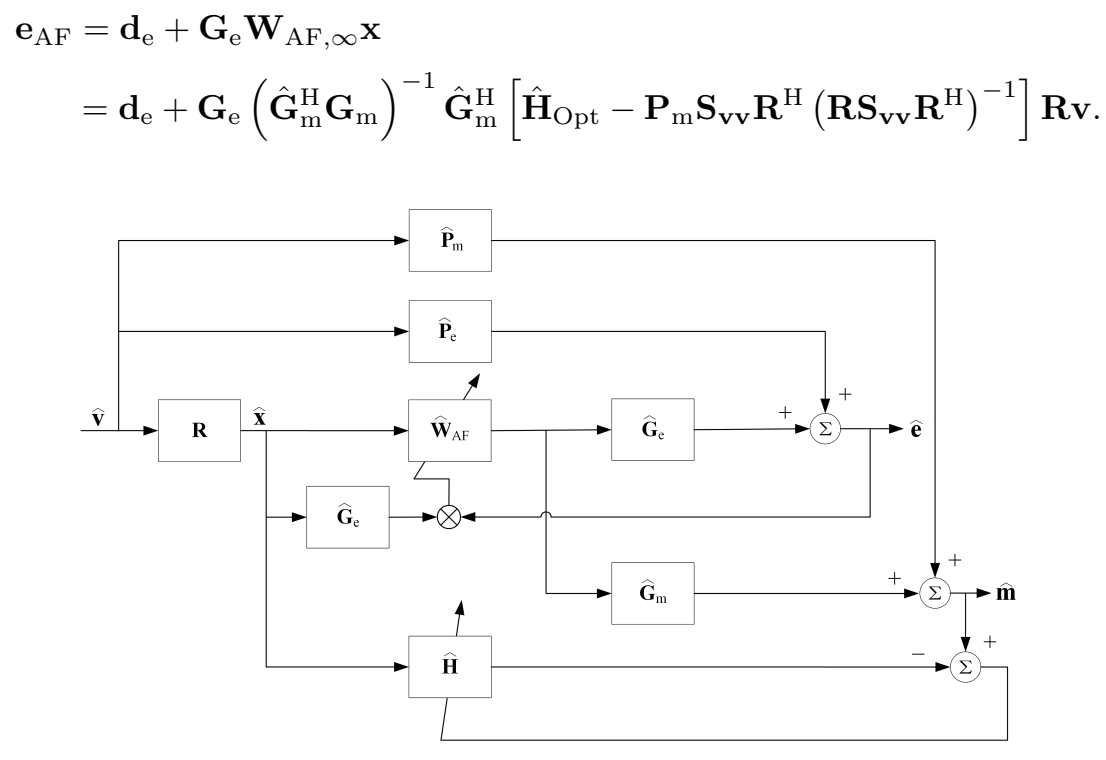

(a)

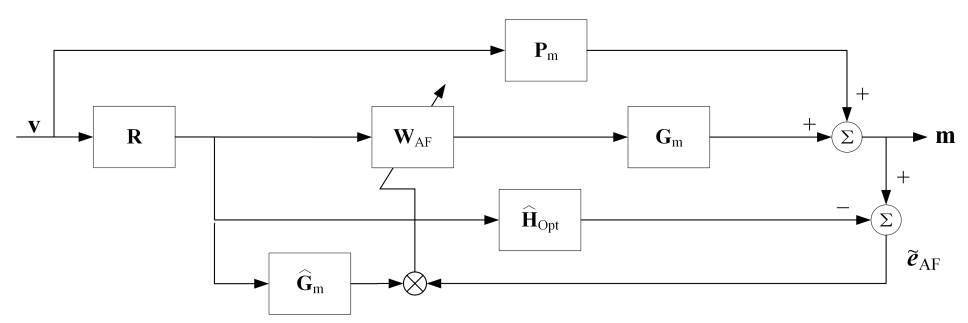

(b)

Figure 2: The block diagram of the AF method: (a) identification phase; (b) control phase.

\section{Application of the virtual sensing methods using measured data}

In this section, the two virtual sensing methods presented in the previous section are applied to data recorded in the cabin of a large SUV as measured and discussed by Jung et al 9]. The SUV was tested on two different road surfaces, referred to as "smooth" and "coarse" in Ref. 8, and for two different speeds, $30 \mathrm{mph}$ and $50 \mathrm{mph}$, for which the road noise was the dominant component of the internal noise in the vehicle. There were in total 14 monitoring microphones mounted in the cabin, attached to the trim panels with duct tape, and two error microphones were located in the ears of a dummy head, as shown in Fig. 3 . Two loudspeakers, which were used as the secondary sources, were placed either sides of the headrest and are positioned 35cm away from each other. In the following simulations, microphones number 1, 2, 13 and 14 in Fig. 3 are used as the monitoring microphones [9], and 8 reference microphones signals were were obtained 
from transducers placed close to suspensions of the vehicle, to measure the components of the road noise that constitute the primary field to be controlled in this case used to measure the primary field. The plant responses for the secondary sources to the error and monitoring microphones were measured during the initial identification phase when the car was stationary. By firmly installing the dummy head and the loudspeakers, the plant responses of the secondary paths are assumed to be unchanged during the driving measurements. The details of the disturbances and the plant responses used in this section can be found in Ref. [9].

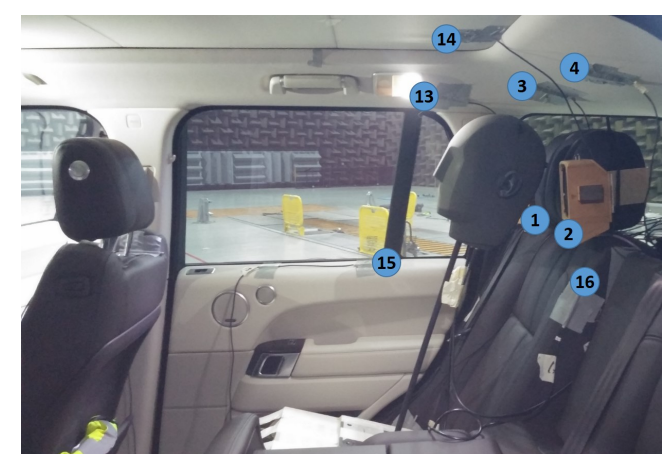

(a)

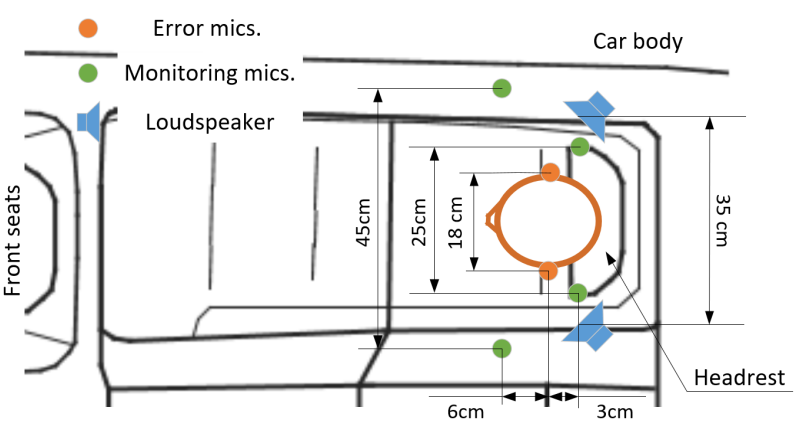

(b)

Figure 3: The installation of the microphones inside the cabin of the test car: (a) on-site view, (b) plan view. Microphones No. 1, 2, 13 and 14 were used as the monitoring microphones and the error microphones are located in the ears of the dummy head $[9]$.

\subsection{Performance under the nominal condition}

In this section, the set of data measured on the coarse road at a car speed of $50 \mathrm{mph}$ has been used to calculate the filters $\hat{\mathbf{H}}_{\mathrm{Opt}}$ and $\hat{\mathbf{O}}_{\mathrm{Opt}}$. The same set of data is then used to evaluate the control performance. In this case, all plant responses are identical for both the identification phase and the control phase. This situation, where the road condition and all plant responses are unchanged, is defined as the nominal condition.

Fig. 4 (4) shows the spectrum of the A-weighted sound pressure levels (SPL) at the error microphones both before control and also after active control using each of the two virtual sensing methods. Above about $600 \mathrm{~Hz}$, the AF method has slightly superior performance compared to the RM method, in which $\hat{\mathbf{O}}_{\text {Opt }}$ has been calculated without regularisation. Fig. 44(b) shows the corresponding levels of attenuation $L_{\text {att }}$, which is defined by

$$
L_{\text {att }}=-10 \log _{10}\left[\operatorname{Tr}\left(\mathbf{S}_{\mathbf{e e}}\right) / \operatorname{Tr}\left(\mathbf{S}_{\mathbf{d}_{\mathrm{e}} \mathbf{d}_{\mathrm{e}}}\right)\right]
$$

in which $\mathbf{S}_{\mathbf{e e}}$ and $\mathbf{S}_{\mathbf{d}_{\mathrm{e}} \mathbf{d}_{\mathrm{e}}}$ are the respective power spectral density matrices of the overall error, $\mathbf{e}$, and the primary disturbance, $\mathbf{d}_{\mathrm{e}}$. The level of the estimation error for the RM method, as defined in Eq. (6), is also shown, which limits the performance of the RM method above $600 \mathrm{~Hz}$. Below this frequency, the performance of both control signals is determined by the limited coherence between the reference signals and the microphone signals. 


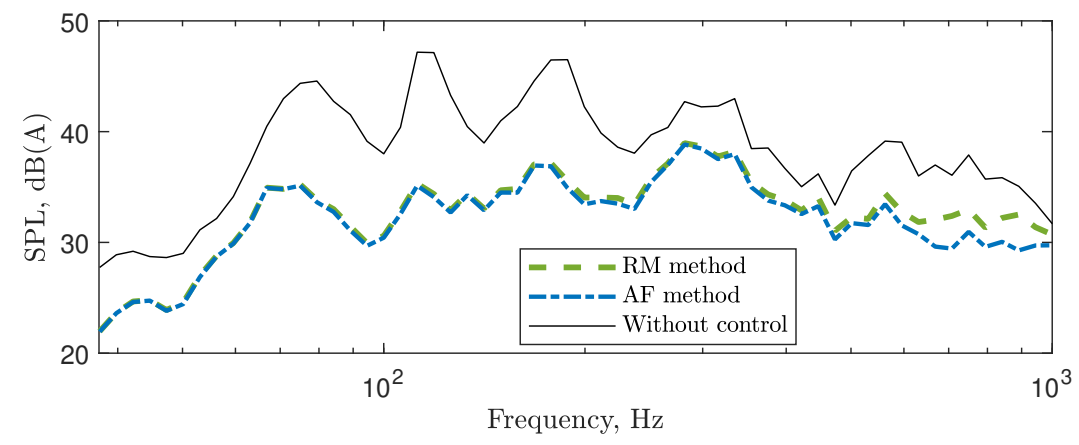

(a)

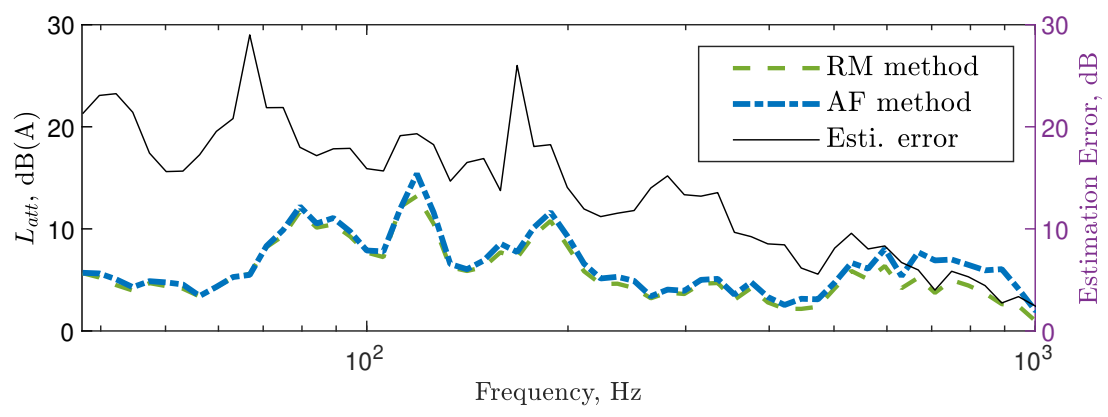

(b)

Figure 4: The 1/12 octave spectra of: (a) the A-weighted sound pressure level (SPL) at the error microphones with and without ANC using each of the two the virtual sensing methods; (b) the corresponding levels of noise attenuation with the additional solid line showing the estimation error of the RM method. The original uncontrolled spectrum, i.e. the solid line in (a), is recorded by running the test car on a coarse road at a speed of $50 \mathrm{mph}$. The same data set is used for identification phase of both virtual sensing methods. 


\subsection{Performance with different reference signals} of the car changes in some way. For instance, changes could be introduced by movement of the listener's seat or simply the presence of another object in the cabin. such changes inevitably lead to changes in the plant responses. With the help of other assisting system, for example the head-tracking system presented in Ref. [15], the two internal filters $\mathbf{O}_{\text {Opt }}$ and $\hat{\mathbf{H}}_{\text {Opt }}$ could be

For practical applications, to maximise the attenuation performance, it is best that the primary field during the identification phase is the same as, or at least similar to, that measured during the control phase, for training the filters of $\mathbf{O}_{\mathrm{Opt}}$ and $\hat{\mathbf{H}}_{\mathrm{Opt}}$. In practice, however, the reference signals used to train the virtual sensing filters will usually differ from those used at the control phase. To investigate this scenario, the training set used to identify $\hat{\mathbf{O}}_{\text {Opt }}$ and $\hat{\mathbf{H}}_{\text {Opt }}$ is altered to the smooth road conditions and other speeds.

Fig. 5 shows the A-weighted SPL at the error microphones before and after control using the two virtual sensing methods using three different sets of measured data in the identification phase, but with the control phase implemented using the $50 \mathrm{mph}$ coarse road data. No regularisation is used in the generation of the RM results presented in this figure. In Fig. 5(a), when the training road condition is changed to smooth, but the vehicle speed remains at $50 \mathrm{mph}$, the spectrum of the RM method is similar to its counterpart given in Fig. 4. The higher performance of the AF method above $600 \mathrm{~Hz}$, seen in Fig. 4(a), is, however, no longer observed. In Figs. 5(b) and (c) show the results obtained when the training sets were measured at a lower speed of $30 \mathrm{mph}$ on the coarse and smooth roads. For the RM method, the lower operation speed does not cause significant reduction in performance, especially at low frequencies, although a small decrease in performance of around $1 \mathrm{~dB}$ can be seen when the frequency is above $600 \mathrm{~Hz}$. For the AF method, however, the noise reduction is significantly reduced compared to the RM method across the entire frequency range.

To further highlight the changes in performance caused by a difference in the reference signals, Table 1 shows a summary of $\bar{L}_{\text {att }}$, the levels of attenuation in the mean square pressure averaged over the full bandwidth of the measurement. When the reference signals used at the identification phase are the same as the ones used during the control phase, the AF method is predicted to provide an averaged attenuation level of around $7.2 \mathrm{~dB}$, which is $0.4 \mathrm{~dB}$ higher than that of the RM method. When the data set for the smooth road with a speed of $50 \mathrm{mph}$ is used for training, however, $\bar{L}_{\text {att,AF }}$ drops to $6.3 \mathrm{~dB}$ and the advantage over the RM method is lost, which has an $\bar{L}_{\text {att,RM }}$ of $6.6 \mathrm{~dB}$. When training data is used from the coarse and smooth roads at $30 \mathrm{mph}, \bar{L}_{\text {att,RM }}$ falls by only $0.2 \mathrm{~dB}$, where $\bar{L}_{\mathrm{att}, \mathrm{AF}}$, drops down by another $1.2 \mathrm{~dB}$ or $1.3 \mathrm{~dB}$. In general, the RM method that displays better performance than the AF method when the reference signals change.

\subsection{Performance with changes in the plant responses}

In addition to changes in the disturbance, it is quite common that the interior environment updated with the changes in the environment, to achieve a new nominal performance. However, 


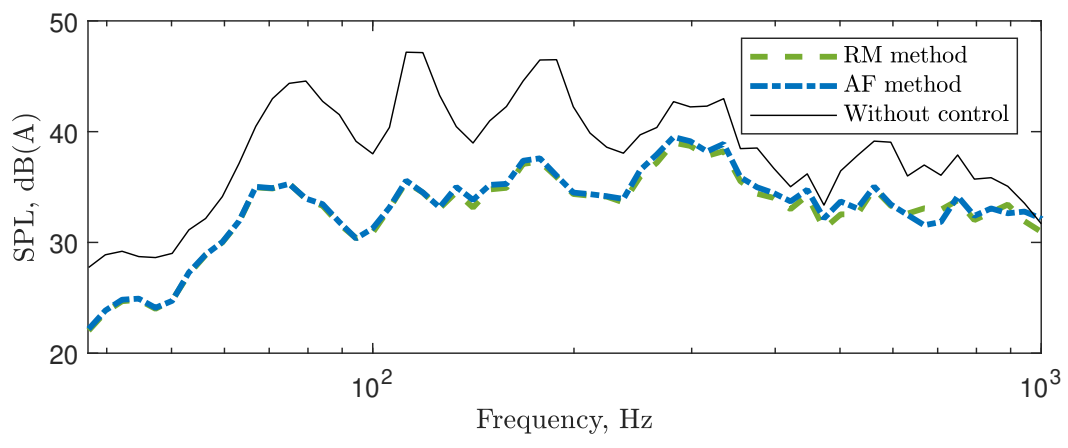

(a) trained by using data set of smooth road, $50 \mathrm{mph}$

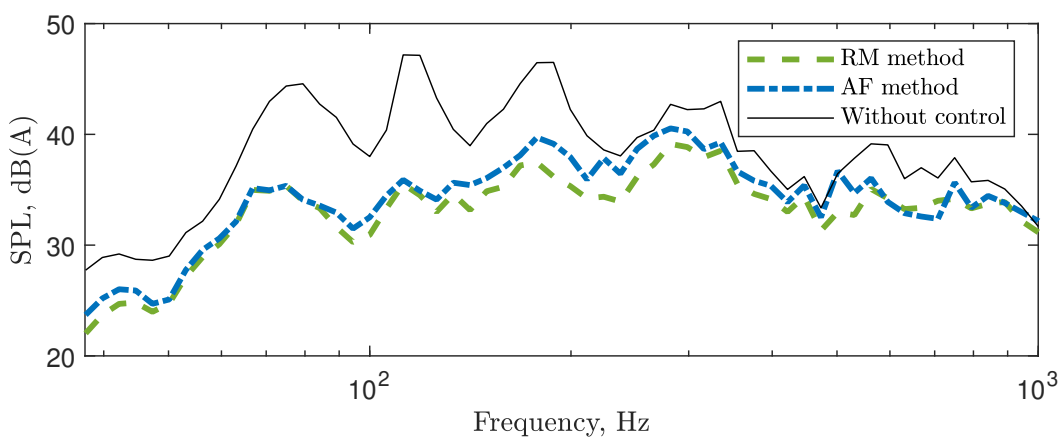

(b) trained by using data set of coarse road, $30 \mathrm{mph}$

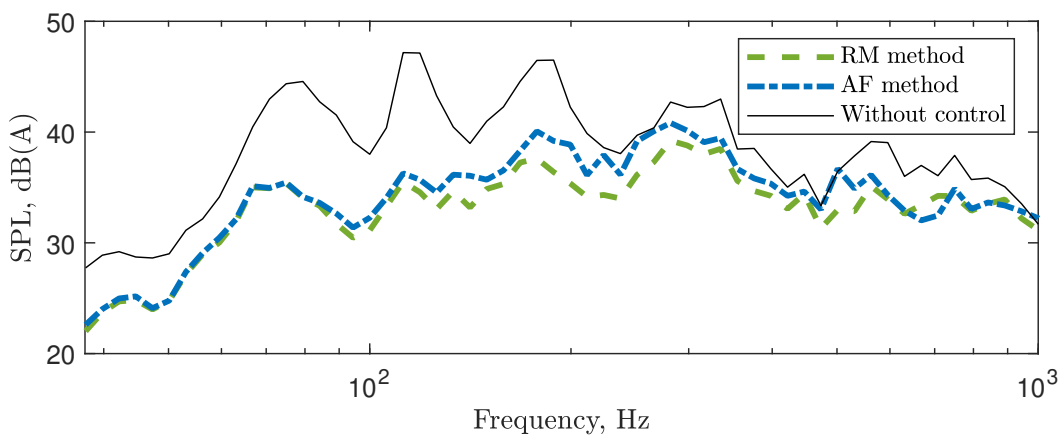

(c) trained by using data set of smooth road, $30 \mathrm{mph}$

Figure 5: The 1/12 octave Spectra of the A-weighted sound pressure level (SPL) at the error microphones with and without active control using the virtual sensing methods. The original spectrum (solid line) is recorded by running the test car on a coarse road with a speed of $50 \mathrm{mph}$. The identification phase for the virtual sensing techniques, however, were carried and using the data sets measured in the same test car under different road conditions and speeds. 
Table 1: Summary of the levels of attenuation $\bar{L}_{\text {att }}$ of the three virtual sensing methods averaged over $1 / 12$ octave band obtained by using different measured data for training and the coarse road at 50mph for control.

\begin{tabular}{lcc}
\hline Training set & $\bar{L}_{\text {att, RM }}, \mathrm{dB} \bar{L}_{\mathrm{att}, \mathrm{AF}}, \mathrm{dB}$ \\
\hline Coarse road, 50 mph & 6.8 & 7.2 \\
(Nominal) & & \\
Smooth road, 50 mph & 6.6 & 6.3 \\
Coarse road, 30 mph & 6.4 & 5.1 \\
Smooth road, 30 mph & 6.4 & 5.0 \\
\hline
\end{tabular}

when the changes cannot be accurately measured, they will influence the attenuation performance. To investigate the effect of changes in the plant responses in the performance of the two virtual sensing methods, a second set of plant response measurements were utilised [16], measured with an in-car ANC system with a similar loudspeaker/microphone layout to that shown in Fig. 3 . These additionally-measured plant responses were acquired under several different conditions that caused perturbations in the acoustic responses. These measured plant changes were then added to the original plant responses to investigate the influence of changes in the plant responses $\mathbf{G}_{\mathrm{m}}$ and $\mathbf{G}_{\mathrm{e}}$ on the control performance of the two virtual sensing methods.

Figs. 6(a) and 6(b) show the SPL of the two virtual sensing methods after these perturbations are introduced in $\mathbf{G}_{\mathrm{m}}$ and $\mathbf{G}_{\mathrm{e}}$ respectively. The regularisation factor for the RM method $\beta_{\mathrm{RM}}$ is initially set to 0 . The spectra under the nominal condition for both methods are also given for comparison. In Fig. 6, the perturbation in $\mathbf{G}_{\mathrm{m}}$ causes significant deterioration in the performance of the two methods at frequencies below around $100 \mathrm{~Hz}$. Fig. 6(b), where the perturbation of $\mathbf{G}_{\mathrm{e}}$ is introduced, similarly shows obvious attenuation reductions for both methods. The RM method that does not perform as well as the AF method for perturbation in $\mathbf{G}_{\mathrm{m}}$, whereas the two methods behave in a similar way for the considered perturbation in $\mathbf{G}_{\mathrm{e}}$.

The robustness of the RM method to changes in plant response could be improved by including regularisation in the design of the observation filter, as will be discussed in Sec. 4.4 .

\section{Theoretical performance and numerical simulations of an idealised headrest system}

The results obtained based on the measurements indicate that the two virtual sensing methods respond differently under different conditions. Therefore, numerical simulations for an idealised geometry are conducted to investigate the causes of these differences and provide additional insight.

The loudspeaker and microphone configuration used for the simulations is shown in Fig. 7. The dimensions in the figure are normalised by a reference length $L$, which is the distance between the virtual error microphones, which is set to $0.15 \mathrm{~m}$ in the simulations. A normalised frequency $k L$ is defined by the wavenumber $k=\omega / c$ and the reference length $L$, where $k L$ is also equal to $2 \pi$ times $L$ divided by the wavelength. Therefore, $k L=0.28$ corresponds to $100 \mathrm{~Hz}$ and $k L=2.8$ 


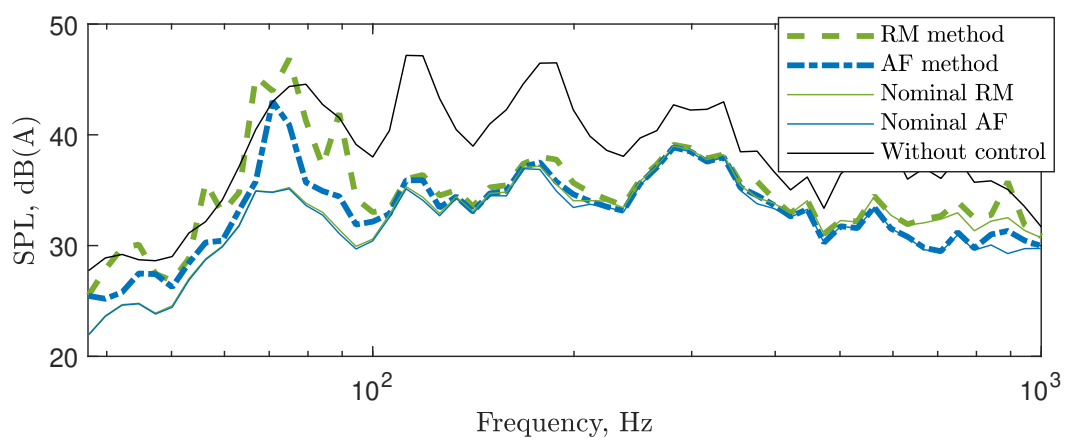

(a)

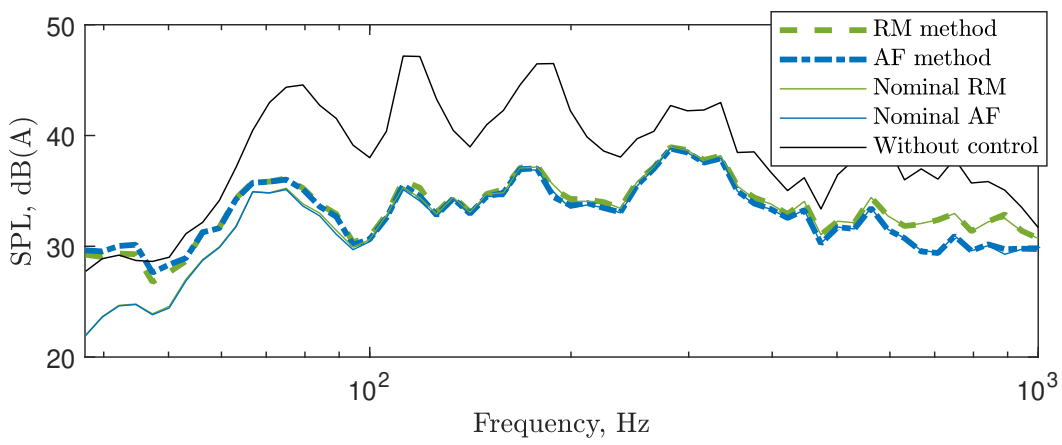

(b)

Figure 6: The 1/12 octave band spectra of the two virtual sensing methods with and without the perturbations in the plant responses of (a) $\mathbf{G}_{\mathrm{m}}$ and (b) $\mathbf{G}_{\mathrm{e}}$. The two methods are trained and tested on the $50 \mathrm{mph}$ coarse road data. The regularisation is not applied for the RM method. 
corresponds to $1 \mathrm{kHz}$. This configuration resembles the headrest ANC system implemented for the in-car measurements. It consists of 2 secondary sources and 4 monitoring microphones and 2 error microphones that are symmetrically distributed about the y-axis. An approximation to a diffuse field is used as the primary field for both identification and control. This field is realized by 491 uncorrelated monopoles with uniform source strength, $\mathbf{v}$, evenly distributed on the surface of a sphere of diameter of $1.5 \mathrm{~m},\left[10\right.$, 17, which ensures that the power spectral matrices of $\mathbf{S}_{\hat{\mathbf{v}} \hat{\mathbf{v}}}$ and $\mathbf{S}_{\mathbf{v v}}$, above, are identity matrices.

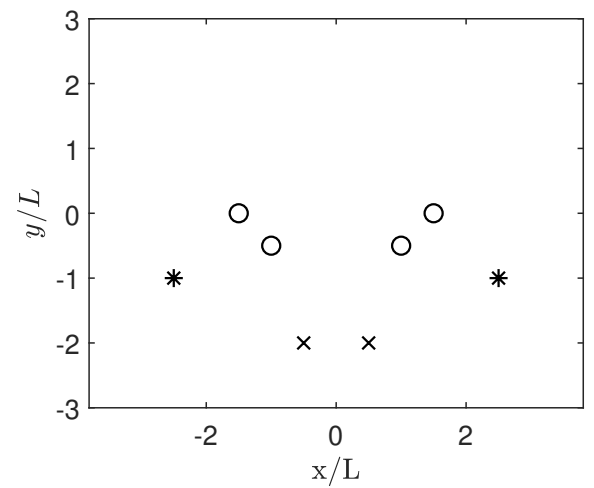

Figure 7: The loudspeaker and microphone configurations used for the virtual sensing methods: *, the secondary sources; $\circ$, the monitoring microphones; $\times$, the virtual error microphones.

\subsection{Nominal condition}

The nominal condition is considered first, where all the plant responses are identical in the control and the identification phases. By assuming the reference signals perfectly represent the primary sources $\hat{\mathbf{R}}=\mathbf{R}=\mathbf{I}$ in Figs. 1 and 2 , the nominal responses for the two virtual sensing methods, following Eqs. (13) and (23), are simply

$$
\mathbf{e}_{0, \mathrm{RM}}=\mathbf{d}_{\mathrm{e}}-\mathbf{G}_{\mathrm{e} 0}\left[\mathbf{G}_{\mathrm{e} 0}^{\mathrm{H}} \mathbf{G}_{\mathrm{e} 0}\right]^{-1} \mathbf{G}_{\mathrm{e} 0}^{\mathrm{H}} \hat{\mathbf{O}}_{\mathrm{Opt}} \mathbf{d}_{\mathrm{m}}
$$

and

$$
\mathbf{e}_{0, \mathrm{AF}}=\mathbf{d}_{\mathrm{e}}-\mathbf{G}_{\mathrm{e} 0}\left[\mathbf{G}_{\mathrm{e} 0}^{\mathrm{H}} \mathbf{G}_{\mathrm{e} 0}\right]^{-1} \mathbf{G}_{\mathrm{e} 0}^{\mathrm{H}} \mathbf{d}_{\mathrm{e}}
$$

where the subscript $(\cdot)_{0}$ denotes the nominal condition. Since, as shown in Fig. 7 the number of secondary sources is equal to the number of the error microphones, the term $\mathbf{G}_{\mathrm{e} 0}\left[\mathbf{G}_{\mathrm{e} 0}^{\mathrm{H}} \mathbf{G}_{\mathrm{e} 0}\right]^{-1} \mathbf{G}_{\mathrm{e} 0}^{\mathrm{H}}$ becomes the identity matrix, which further simplifies the nominal responses. $\mathbf{e}_{0, \mathrm{RM}}$ is then identical to the expression for the estimation error in Eq. (6), whereas, $\mathbf{e}_{0, \mathrm{AF}}$ becomes zero, which indicates an infinite level of attenuation for the AF method at the error microphones.

Fig. 8 shows the estimation error, and hence the attenuation for the RM method under nominal condition, using the configuration shown in Fig. 7 at different frequencies. No regularisation was used in the calculation of $\hat{\mathbf{O}}_{\text {Opt }}$ here. The level of attenuation gradually decreases with increasing of the normalised frequency $k L$, which is an indication of the deterioration in the spatial correlation 
between the microphones, as explained in Ref. [5]. This is consistent with the measurement result in Fig. 4(b) and since the AF method is theoretically capable of infinite attenuation at the error microphones, it is clearly superior to the RM method in terms of the attenuation under nominal conditions.

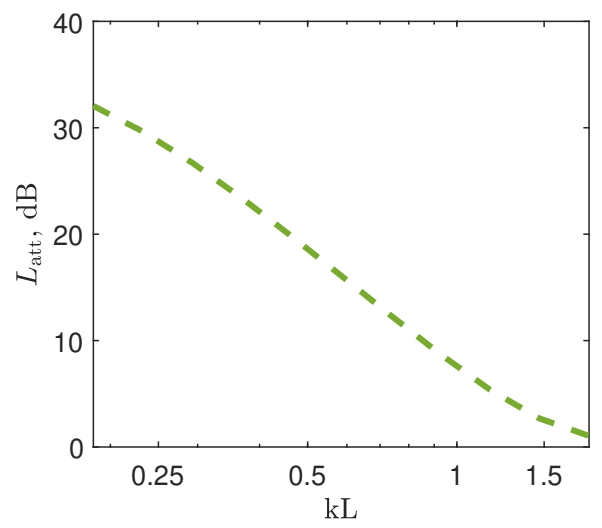

Figure 8: The nominal attenuation of the RM method for the configuration shown in Fig. 7. which is due to the estimation error.

Fig. 9 shows the spatial distribution of the attenuation in the mean square level of the primary field over the whole space shown in Fig. 7, to further compare the two virtual sensing methods under the nominal condition. These results show that, at low frequencies, the two methods have very similar attenuation distribution away from the immediate vicinity of the error microphone. At higher frequencies, the AF method achieves better performance compared to the RM method, especially around the locations of the error microphones.

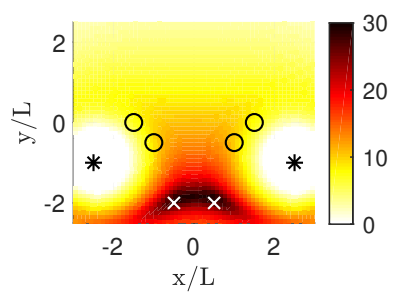

(a) RM, $k L=0.25$

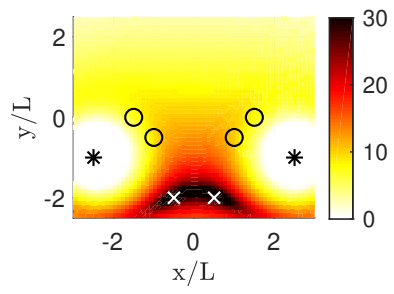

(d) $\mathrm{AF}, k L=0.25$

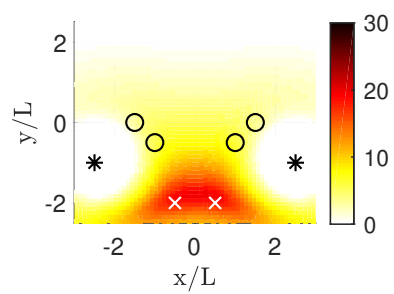

(b) RM, $k L=0.5$

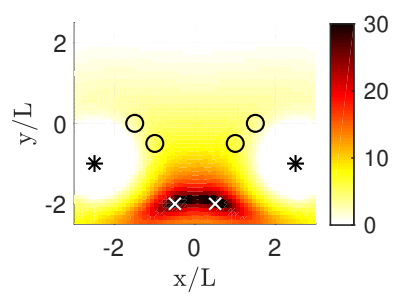

(e) $\mathrm{AF}, k L=0.5$

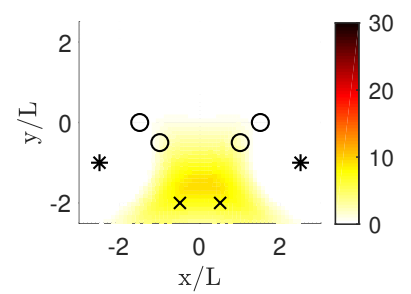

(c) $\mathrm{RM}, k L=1$

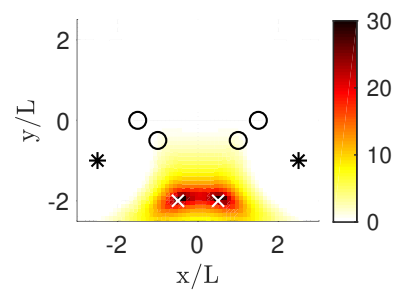

(f) $\mathrm{AF}, k L=1$

Figure 9: The spatial distribution of $L_{\mathrm{att}, \mathrm{AF}}$ and $L_{\mathrm{att}, \mathrm{RM}}$ under the nominal condition at different frequencies. From left to right, $k L=0.25,0.5,1$. 
In practice, an imperfect coherence between the reference and the error signals will affect the attenuation performance of both virtual sensing methods, as noted in Sec 3.1. This phenomenon is modelled by using an incomplete set of reference signals in this section.

The incomplete set of reference signals can be simulated by modifying $\mathbf{R}$, the response from the primary sources to the reference signals under nominal condition in Figs. 1 and 2 which is assumed to have a size of $N \times N$, and to be of the form

$$
\mathbf{R}=\left[\begin{array}{cc}
\mathbf{R}_{\mathrm{c}} & \mathbf{0} \\
\mathbf{0} & \mathbf{R}_{\mathrm{u}}
\end{array}\right]
$$

where $\mathbf{R}_{\mathrm{c}}$ denotes the paths of the primary sources that are perfectly represented by the reference microphones, where $\mathbf{R}_{\mathrm{c}}=\mathbf{I}^{(K \times K)}$ and $K$ denotes the number of the primary sources that can be accurately represented. On the other hand, $\mathbf{R}_{\mathrm{u}}$ denotes the plants of the primary sources that are not perfectly represented by the reference signals. For simplicity, it is assumed that $\mathbf{R}_{\mathrm{u}}=\mathbf{0}$, and therefore, no knowledge of those primary sources is available. The primary sources that are not captured by the reference microphones will thus not be attenuated by the feedforward control system, but will contribute directly to the virtual error microphones, which, consequently, will deteriorate the attenuation performance achieved by the virtual sensing control methods. Assuming that each primary source contributes equally to the mean square disturbance, a theoretical attenuation can then be obtained as

$$
L_{\text {att }}=10 \log _{10} \frac{N}{N-K}
$$

where $N$ is the number of primay sources with equal strength and $K$ is the number of reference signals.

Fig. 10(a) shows a comparison between the theoretical attenuation given by Eq. (28) and the attenuations achieved by simulating the two virtual sensing methods. According to this figure, the AF method matches the theoretical result perfectly. Whereas, the RM method has slightly lower levels of attenuation, due to the contribution of the estimation error at high frequencies. This is demonstrated more clearly in Fig. 10(b), which shows the attenuations for the two virtual sensing methods as a function of normalised frequency, $k L$, where a quarter of the primary sources are not captured by the reference microphones. The estimation error for the RM method is also shown. Clearly, at low frequencies, where the estimation error is small, the incomplete set of references signals determines the performance limits for both the RM method and the AF method in a very similar way. The attenuation is around $6 \mathrm{~dB}$ in both cases, which corresponds to the theoretical calculation provided by Eq. 28). At high frequencies, where the estimation error for the RM method gradually increases, the attenuation achieved by the RM method is determined by the estimation error and hence falls off. Similar features to those described here can also be found for the results predicted from the in-car measurements in Fig. 4 (b). 


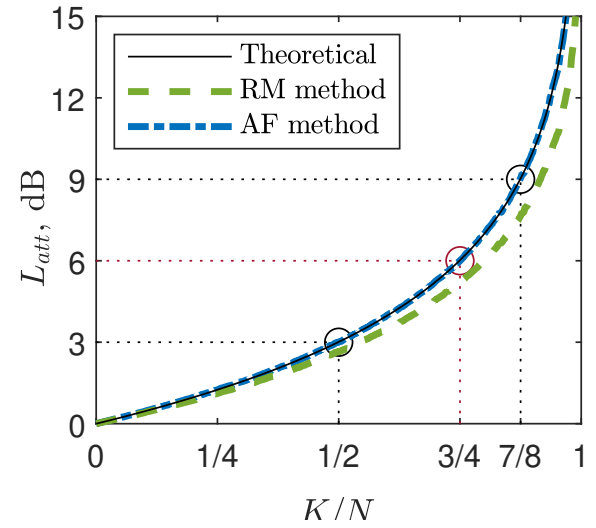

(a)

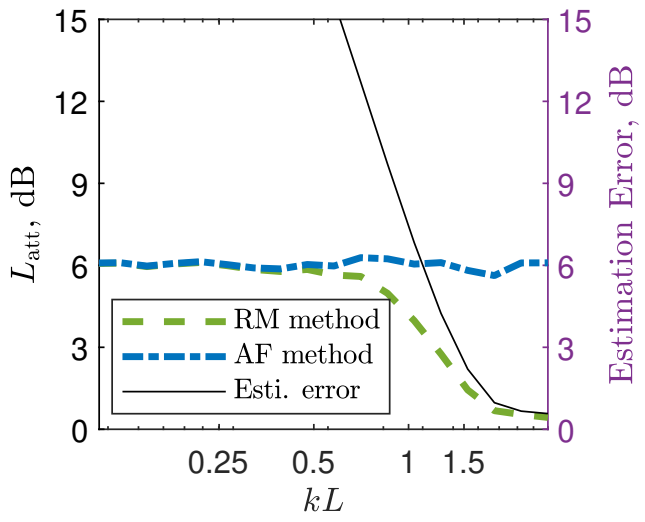

(b)

Figure 10: Attenuations for the two virtual sensing strategies with incomplete reference signals: (a) attenuation with only $K$ reference signals but $N$ primary signals; (b) the attenuation spectra obtained from simulation of the two virtual sensing methods when $K / N=3 / 4$.

\subsection{Error due to uncertainties in the reference signals}

Changes in the statistical properties of the reference signals can be simulated by introducing uncertainties into the response, $\mathbf{R}$, between the secondary sources and the reference signals. This would be similar to the changes in the vehicle reference signals discussed in Sec. 3.2, which might arise from changes in the road or driving conditions. It is assumed that during the identification phase, $\hat{\mathbf{R}}=\mathbf{R}_{0}$, and in the control phase, a perturbation matrix $\Delta \mathbf{R}$ is added to $\hat{\mathbf{R}}$. The perturbation matrix $\Delta \mathbf{R}$ is assumed to be unstructured [17]. The unstructured uncertainty of $\Delta \mathbf{R}$ has zero mean and is uncorrelated with the corresponding nominal responses [17, and so

$$
\begin{gathered}
E[\Delta \mathbf{R}]=\mathbf{0} \\
E\left[\Delta \mathbf{R}^{\mathrm{H}} \mathbf{R}_{0}\right]=\mathbf{0} .
\end{gathered}
$$

The elements of $\Delta \mathbf{R}$ are also assumed to be mutually uncorrelated, and satisfy

$$
E\left[\Delta \mathbf{R}^{\mathrm{H}} \Delta \mathbf{R}\right]=\gamma_{\Delta \mathbf{R}} \mathbf{I}
$$

where $\gamma_{\Delta \mathbf{R}}$, the power of the uncertainty $\Delta \mathbf{R}$, is given by

$$
\gamma_{\Delta \mathbf{R}}=\frac{1}{N} \varepsilon_{\Delta \mathbf{R}}^{2}\left\|\mathbf{R}_{0}\right\|_{\mathrm{F}}^{2},
$$

where $\varepsilon_{\Delta \mathbf{R}}$ is the level of relative uncertainty. This parameter can be defined as

$$
\varepsilon_{\Delta \mathbf{R}}^{2}=\|\Delta \mathbf{R}\|_{\mathrm{F}}^{2} /\left\|\mathbf{R}_{0}\right\|_{\mathrm{F}}^{2} .
$$

The properties defined in Eqs. 29) to (33) are realised by modelling the real and imaginary parts of $\Delta \mathbf{R}$ respectively by using the function 'randn' embedded in MATLAB. Enough samples are 
required for the numerical modelling of the unstructured uncertainties to satisfy the properties given previously. Here, 250 realisations are used to obtain the expectation values.

By introducing $\Delta \mathbf{R}$ to both of the virtual sensing methods, the overall error signal $\mathbf{e}$ is given by the superposition of the nominal response $\mathbf{e}_{0}$ and the error caused due to $\Delta \mathbf{R}, \mathbf{e}_{\text {uncert }}$. The expressions of $\mathbf{e}_{\text {uncert }}$ for the two virtual sensing methods can be derived, based on Eqs. (13) and (23), as

$$
\begin{gathered}
\mathbf{e}_{\text {uncert }, \mathrm{RM}}=-\hat{\mathbf{O}}_{\mathrm{Opt}} \mathbf{P}_{\mathrm{m} 0} \Delta_{\mathbf{R}} \mathbf{v} \\
\mathbf{e}_{\text {uncert }, \mathrm{AF}}=-\left(\mathbf{G}_{\mathrm{e} 0}\left[\mathbf{G}_{\mathrm{m} 0}^{\mathrm{H}} \mathbf{G}_{\mathrm{m} 0}\right]^{-1} \mathbf{G}_{\mathrm{m} 0}^{\mathrm{H}} \mathbf{P}_{\mathrm{m} 0}-\mathbf{P}_{\mathrm{e} 0}\right) \Delta \mathbf{R} \mathbf{v} \\
-\mathbf{G}_{\mathrm{e} 0}\left[\mathbf{G}_{\mathrm{m} 0}^{\mathrm{H}} \mathbf{G}_{\mathrm{m} 0}\right]^{-1} \mathbf{G}_{\mathrm{m} 0}^{\mathrm{H}} \mathbf{P}_{\mathrm{m} 0} \Delta_{\mathbf{R}} \mathbf{v}
\end{gathered}
$$

where

$$
\Delta_{\mathbf{R}}=\mathbf{R}^{\mathrm{H}}\left(\mathbf{R S}_{\mathbf{v v}} \mathbf{R}^{\mathrm{H}}\right)^{-1} \mathbf{R}-\mathbf{R}_{0}^{\mathrm{H}}\left(\mathbf{R}_{0} \mathbf{S}_{\mathbf{v v}} \mathbf{R}_{0}^{\mathrm{H}}\right)^{-1} \mathbf{R}_{0} .
$$

The elements of $\Delta_{\mathbf{R}}$ are very small, even when $\varepsilon_{\Delta \mathbf{R}}$ is significant, which consequently makes the terms related to $\Delta_{\mathbf{R}}$ become negligible. Therefore, Eqs. 34 and 35 can be approximately given by

$$
\begin{gathered}
\mathbf{e}_{\text {uncert }, \mathrm{RM}}=0 \\
\mathbf{e}_{\text {uncert }, \mathrm{AF}}=-\left(\mathbf{G}_{\mathrm{e} 0}\left[\mathbf{G}_{\mathrm{m} 0}^{\mathrm{H}} \mathbf{G}_{\mathrm{m} 0}\right]^{-1} \mathbf{G}_{\mathrm{m} 0}^{\mathrm{H}} \mathbf{P}_{\mathrm{m} 0}-\mathbf{P}_{\mathrm{e} 0}\right) \Delta \mathbf{R v}
\end{gathered}
$$
changes in the plant responses for the two methods are listed in Table 2 . The numerical simulations 


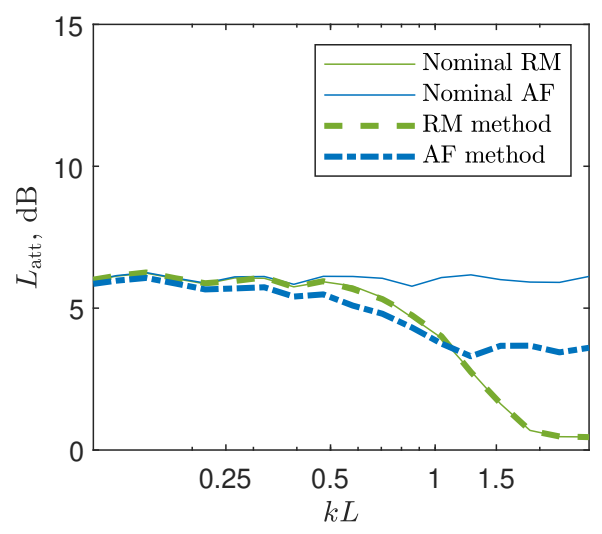

Figure 11: Attenuations for the two virtual sensing strategies with unstructured perturbations in $\mathbf{R}, \varepsilon_{\Delta \mathbf{R}}$ is set to 0.15. The same incomplete reference signals were used as in Fig 10(b)

are shown in Fig. 12 For all numerical simulations presented here, an imperfect reference, with $K / N=3 / 4$, is used to make the simulated results comparable to the measured-data based results in Sec. 3. $\varepsilon$ is again set to 0.15 for each case. All the results in Fig. 12 are shown both with and without regularisation of the observation filter in the RM method, as in Eq. (3). The regularisation factor, $\beta_{\mathrm{RM}}$, is set to 0.2 at frequencies below $k L=0.9$ and then gradually decreases to 0 at higher frequencies.

In Table 2 , the perturbation in $\Delta \mathbf{G}_{\mathrm{m}}$ is included in $\Delta_{\mathbf{G}_{\mathrm{m}}, \mathrm{RM}}$ and $\Delta_{\mathbf{G}_{\mathrm{m}}, \mathrm{AF}}$. Fig. 12(a) shows the simulated results for uncertainties in $\mathbf{G}_{\mathrm{m}}$ and it can be seen that the RM method is most significant affected when no regularisation is used in the observation filter. The essential difference between the two methods is that $\Delta \mathbf{G}_{\mathrm{m}}$ is multiplied by the observation filter for the RM method, whereas it is directly proportional for the AF method. Jung et al [5] show that increasing the regularisation factor will reduce the effect of the condition number of $\mathbf{S}_{\hat{\mathbf{d}}_{\mathrm{m}} \hat{\mathbf{d}}_{\mathrm{m}}}$ in Eq. (3), which makes the RM method robust against perturbations. The simulation results for unstructured perturbation in $\mathbf{P}_{\mathrm{m}}$ are shown in Fig. 12(b). Once again, the attenuation is more compromised for the RM method than for the AF method, although appropriate regularisation improves the RM results. The simulation results are again well predicted by the theoretical results in Table 2 , in which the perturbation for the RM method is again multiplied by the observation filter and so is sensitive to its regularisation. Figs. $12(\mathrm{c})$ and $12(\mathrm{~d})$ show that the attenuations with unstructured perturbations in $\mathbf{G}_{\mathrm{e}}$ and $\mathbf{P}_{\mathrm{e}}$ are far less affected than for perturbations in $\mathbf{G}_{\mathrm{m}}$ and $\mathbf{P}_{\mathrm{m}}$, for both virtual sensing methods. These results are well predicted by the theoretical results in Tabel 2 , where it can be seen that the effect of perturbations in $\mathbf{P}_{\mathrm{e}}$ are predicted to be small, but the same, for both RM and AF methods.

In general, the AF method is more robust against perturbations in the plant responses than the RM method. For the AF method, when the perturbations are relatively small, the errors caused by these perturbations are insignificant compared to the errors introduced by the incomplete references. The RM method is susceptible to perturbations in $\mathbf{G}_{\mathrm{m}}$ and $\mathbf{P}_{\mathrm{m}}$, unless appropriate regularisation is used. 


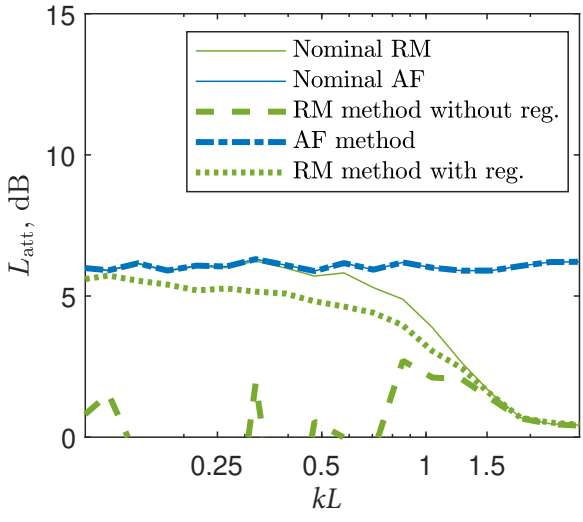

(a) $\Delta \mathbf{G}_{\mathrm{m}}$

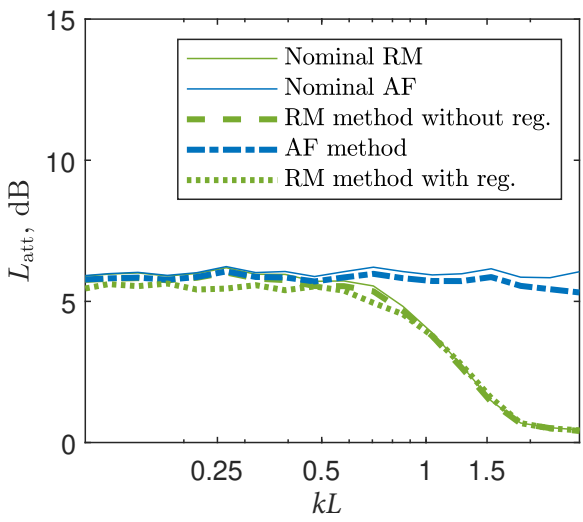

(c) $\Delta \mathbf{G}_{\mathrm{e}}$

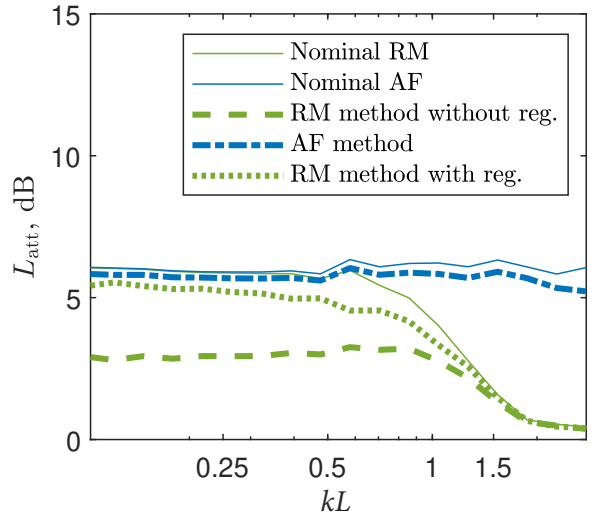

(b) $\Delta \mathbf{P}_{\mathrm{m}}$

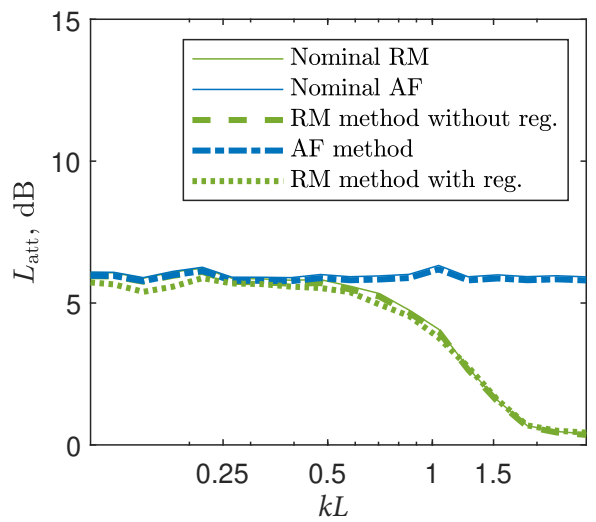

(d) $\Delta \mathbf{P}_{\mathrm{e}}$

Figure 12: Attenuations for the two virtual sensing strategies with unstructured perturbations in (a) $\Delta \mathbf{G}_{\mathrm{m}}$, (b) $\Delta \mathbf{P}_{\mathrm{m}}$, (c) $\Delta \mathbf{G}_{\mathrm{e}}$ and (d) $\Delta \mathbf{P}_{\mathrm{e}} \cdot \varepsilon=0.15$ is used for all four perturbations, and incomplete reference signals, as in Fig. 10(b) were again assumed. 


\begin{tabular}{lll}
\hline Perturbation Method & $\mathbf{e}_{\text {uncert }}$ \\
\hline & RM & $-\Delta_{\mathbf{G}_{\mathrm{m}}, \mathrm{RM}} \hat{\mathbf{O}}_{\mathrm{Opt}} \mathbf{d}_{\mathrm{m}}$, \\
& & where $\Delta_{\mathbf{G}_{\mathrm{m}}, \mathrm{RM}}=\mathbf{G}_{\mathrm{e} 0}\left[\mathbf{G}_{\mathrm{e} 0}^{\mathrm{H}}\left(\mathbf{G}_{\mathrm{e} 0}+\hat{\mathbf{O}}_{\mathrm{Opt}} \Delta \mathbf{G}_{\mathrm{m}}\right)\right]^{-1} \mathbf{G}_{\mathrm{e} 0}^{\mathrm{H}}-\mathbf{I}$ \\
& & $-\left(\mathbf{G}_{\mathrm{e} 0} \Delta_{\mathbf{G}_{\mathrm{m}}, \mathrm{AF}}\left[\mathbf{G}_{\mathrm{e} 0}^{\mathrm{H}} \mathbf{G}_{\mathrm{e} 0}\right]^{-1} \mathbf{G}_{\mathrm{e} 0}^{\mathrm{H}}\right) \mathbf{d}_{\mathrm{e}}$, \\
& $\mathrm{AF}$ & where $\Delta_{\mathbf{G}_{\mathrm{m}}, \mathrm{AF}}=\left[\mathbf{G}_{\mathrm{m} 0}^{\mathrm{H}}\left(\mathbf{G}_{\mathrm{m} 0}+\Delta \mathbf{G}_{\mathrm{m}}\right)\right]^{-1} \mathbf{G}_{\mathrm{m} 0}^{\mathrm{H}} \mathbf{G}_{\mathrm{m} 0}-\mathbf{I}$ \\
& $\mathrm{RM}$ & $-\hat{\mathbf{O}}_{\mathrm{Opt}} \Delta \mathbf{P}_{\mathrm{m}} \mathbf{v}$ \\
$\Delta \mathbf{P}_{\mathrm{m}}$ & $\mathrm{AF}$ & $-\left(\mathbf{G}_{\mathrm{e} 0}\left[\mathbf{G}_{\mathrm{m} 0}^{\mathrm{H}} \mathbf{G}_{\mathrm{m} 0}\right]^{-1} \mathbf{G}_{\mathrm{m} 0}^{\mathrm{H}}\right) \Delta \mathbf{P}_{\mathrm{m}} \mathbf{v}$ \\
& $\mathrm{RM}$ & $-\Delta_{\mathbf{G}_{\mathrm{e}}} \hat{\mathbf{O}}_{\mathrm{Opt}} \mathbf{d}_{\mathrm{m}}$ \\
& AF & $-\Delta_{\mathbf{G}_{\mathrm{e}}} \mathbf{d}_{\mathrm{e}}$ \\
& & $\Delta_{\mathbf{G}_{\mathrm{e}}}=\Delta \mathbf{G}_{\mathrm{e}}\left[\mathbf{G}_{\mathrm{e} 0}^{\mathrm{H}} \mathbf{G}_{\mathrm{e} 0}\right]^{-1} \mathbf{G}_{\mathrm{e}}^{\mathrm{H}}$ \\
& & $\Delta \mathbf{P}_{\mathrm{e}} \mathbf{v}$ \\
& $\mathrm{RM}$ & $\Delta \mathbf{P}_{\mathrm{e}} \mathbf{v}$ \\
\hline $\mathbf{P}_{\mathrm{e}}$ & $\mathrm{AF}$ &
\end{tabular}

\section{Conclusions}

This paper explains the fundamental differences between the performance of the RM method and the AF method under various conditions. The difference lies in the different strategies used to estimate the disturbance at the virtual error microphones. The RM method explicitly estimates the disturbance at the virtual microphones from the monitoring microphone signals, by using the observation filter, whereas, the AF method implicitly estimates the disturbance at the virtual microphones from the reference signals by using a reference model.

For a virtual sensing ANC system in a car cabin, such as that described in this paper, the AF method is superior compared to the RM method under nominal conditions, especially at high frequencies. This is due to the estimation error associated with the RM method, which limits its performance and generally increases with frequency. In practice, however, the reference signals cannot exactly represent the primary sources, so that the unrepresented primary sources are not controlled by the ANC system and will increase the error. The simulations show that having incomplete reference signals has a similar impact on the two virtual sensing methods.

The robust performance of the two methods is also quite different, when uncertainties are introduced in the various plant responses between the identification and control phases of the algorithms. For perturbations in the reference signals, the RM method tends to be very robust since the design of the observation filter is not significantly affected by the reference signals. The AF method, however, heavily relies on the form of the reference signals, and deteriorates with an increase in the perturbation, unless the additional filter is scheduled on the characterisation of the measured reference signals, as suggested by Shi et al. [18. Perturbations in the plant responses of 
the primary and secondary paths also introduce different effects for the two virtual sensing methods. All four perturbations have very limited effects for the AF method, which indicates its robustness against changes in these plant responses. For the RM method, the plant responses related to the error microphones also have a small effect on the level of attenuation. However, the RM method is quite sensitive to the plant perturbations related to the monitoring microphones. The analytical formulation demonstrates that the errors due to these two perturbations are multiplied by the observation filter, which contains an inverse of a matrix that is often ill-conditioned. This ill conditioning leads to an increase in the size of the observation filter, and consequently, amplifies the perturbations and deteriorates the attenuation performance of the RM method. The robustness of the RM method can greatly be improved by introducing appropriate regularisation.

Therefore, it can be concluded that the best virtual sensing method to use for active control depends on the details of the application, not just in terms of their nominal performance but also in terms of their robustness. The AF method is preferred when the noise sources to be controlled can be accurately measured, for example, for measurements performed in the laboratory, since the model-reference methods are robust against changes in the primary and secondary paths. For active noise control in an environment where the reference signals can change, however, as in many practical applications, for example the one presented in this paper, the RM method may be the better option, due to its insensitivity to the detailed properties of the reference signals.

\section{Acknowledgement}

The authors gratefully acknowledge the support of the UK Engineering and Physical Sciences 300 Research Council (EPSRC) through the DigiTwin project (grant EP/R006768/1). 


\section{References}

[1] M. Pawelczyk, Adaptive noise control algorithms for active headrest system, Control Engineering Practice 12 (9) (2004) 1101-1112.

[2] D. Moreau, B. Cazzolato, A. Zander, C. Petersen, A review of virtual sensing algorithms for active noise control, Algorithms 1 (2) (2008) 69-99.

[3] D. P. Das, D. J. Moreau, B. Cazzolato, Performance evaluation of an active headrest using the remote microphone technique, Proceedings of Acoustics 2011 (2011) 2-4.

[4] A. Siswanto, C.-Y. Chang, S. M. Kuo, Active noise control for headrests, in: 2015 Asia-Pacific Signal and Information Processing Association Annual Summit and Conference (APSIPA), IEEE, 2015, pp. 688-692.

[5] W. Jung, S. J. Elliott, J. Cheer, Estimation of the pressure at a listener's ears in an active headrest system using the remote microphone technique, The Journal of the Acoustical Society of America 143 (5) (2018) 2858-2869.

[6] J. Garcia-Bonito, S. Elliott, C. Boucher, Generation of zones of quiet using a virtual microphone arrangement, The journal of the Acoustical Society of America 101 (6) (1997) 34983516.

[7] S. J. Elliott, W. Jung, J. Cheer, Head tracking extends local active control of broadband sound to higher frequencies, Scientific reports 8 (1) (2018) 1-7.

[8] A. Roure, A. Albarrazin, The remote microphone technique for active noise control, in: INTER-NOISE and NOISE-CON Congress and Conference Proceedings, Vol. 1999, Institute of Noise Control Engineering, 1999, pp. 1233-1244.

[9] W. Jung, S. J. Elliott, J. Cheer, Local active control of road noise inside a vehicle, Mechanical Systems and Signal Processing 121 (2019) 144-157.

${ }_{325}$ [10] S. J. Elliott, J. Cheer, Modeling local active sound control with remote sensors in spatially random pressure fields, The Journal of the acoustical Society of america 137 (4) (2015) 19361946.

[11] S. J. Elliott, W. Jung, J. Cheer, Causality and robustness in the remote sensing of acoustic pressure, with application to local active sound control, in: ICASSP 2019-2019 IEEE International Conference on Acoustics, Speech and Signal Processing (ICASSP), IEEE, 2019, pp. 8484-8488.

[12] J. Sun, In encyclopedia of systems and control, Springer, London, 2014, Ch. Model reference adaptive control, pp. 1-7. 
[13] D. Shi, B. Lam, W.-s. Gan, Analysis of multichannel virtual sensing active noise control to overcome spatial correlation and causality constraints, in: ICASSP 2019-2019 IEEE International Conference on Acoustics, Speech and Signal Processing (ICASSP), IEEE, 2019, pp. 8499-8503.

[14] S. Elliott, Signal processing for active control, Elsevier, 2000.

[15] W. Jung, S. J. Elliott, J. Cheer, Combining the remote microphone technique with headtracking for local active sound control, The Journal of the Acoustical Society of America 142 (1) (2017) 298-307.

[16] S. Elliott, C. Lai, T. Vergez, J. Cheer, Robust stability and performance of local active control systems using virtual sensing, in: Proceedings of the 23rd International Congress on Acoustics (2019), pp. 61-68.

${ }_{345}$ [17] S. J. Elliott, J. Cheer, J.-W. Choi, Y. Kim, Robustness and regularization of personal audio systems, IEEE Transactions on Audio, Speech, and Language Processing 20 (7) (2012) 21232133.

[18] C. Shi, R. Xie, N. Jiang, H. Li, Y. Kajikawa, Selective virtual sensing technique for multichannel feedforward active noise control systems, in: ICASSP 2019-2019 IEEE International Conference on Acoustics, Speech and Signal Processing (ICASSP), IEEE, 2019, pp. 8489-8493. 